prevalence as well as their associated patient characteristics and effective treatment rates. Few studies investigating treatment patterns of GERD have been conducted. Thus, it is significant to investigate current diagnostic practices and treatment patterns of GERD in China and understand the effectiveness of current standard treatments in real-world settings.

Methods The overall analysis population will consist of patients of all ages who were diagnosed with GERD from January 2015 to December 2017. Only the patient's first treatment trajectory will be counted during this period. Specific analysis population details for diagnosis, treatment, and real-world (RW) treatment efficacy are also outlined separately.

Results The sample size is currently unknown but will be determined based on three years of available GERD patient data within target hospitals' scope. The project sample will be selected across 20 hospitals in China. Data from 20 hospitals will be extracted from hospital electronic system. Variables of interest will cover patient socio-demographic characteristics (age, sex, health plan type, hospital, etc.), diagnostic methods, selected treatment details, selected medical history and medication use prior to and after the index date, and medical records of healing over the analysis period. The primary objective is to investigate current diagnostic practices and treatment patterns of GERD in China, including (1) Proportion and trend of GERD patients in different specialist outpatient clinics (digestive, respiratory, otolaryngology, others). (2) Treatment pattern (the treatment of different specialists, treatment of different types of symptoms, the relationship between symptoms and different endoscopic manifestations) of GERD in clinical practice.

Conclusions This GRAND CHINA STUDY would provide important evidence for current diagnostic practices and treatment patterns of GERD as well as evaluate the real-world outcomes of EE in China.

\section{IDDF2019-ABS-0035 THE EFFICACY OF COMBINING SIMETHICONE AND POLYETHYLENE GLYCOL FOR ADENOMA DETECTION DURING COLONOSCOPY: A SYSTEMATIC REVIEW AND META-ANALYSIS OF RANDOMIZED CONTROLLED TRIALS}

${ }^{1} \mathrm{Hu}$ Zhang*, 'Song-Lin Ma, ${ }^{1}$ Jing Liu, ${ }^{1}$ Man-Lin Huang, ${ }^{1}$ Qi-Long Song, ${ }^{1}$ Sheng-Bin Sun, ${ }^{1}$ Yan Fan, ${ }^{1}$ Min Song, ${ }^{1}$ Heng Zhang, ${ }^{2}$ Hui-Hua Luo, ${ }^{2}$ Chang Zhao. ${ }^{1}$ The Central Hospital of Wuhan, Tongji Medical College, Huazhong University of Science and Technology, China; ${ }^{2}$ The Eighth Hospital of Wuhan, China

\subsection{6/gutjnl-2019-IDDFabstracts. 122}

Background Although several randomized controlled trials (RCTs) have shown that the addition of simethicone to polyethylene glycol (PEG) can improve bowel preparation, whether PEG plus simethicone had a beneficial role in the adenoma detection rate (ADR) during a colonoscopy has yet to be confirmed. The aim of this study was to clarify whether adding simethicone to a PEG solution could improve the effectiveness of the ADR.

Methods The PubMed, EMBASE, and Cochrane Library databases were searched for articles published prior to November 2018. The primary outcomes included the ADR and the polyp detection rate (PDR). The secondary endpoints included the efficacy of the colon preparation, procedure-related parameters, adverse events and willingness to repeat preparation. The odds ratios (ORs) and mean difference (MD) with 95\% confidence intervals (CIs) were pooled for discrete and continuous variables. The subgroup analyses and sensitivity analyses were performed.

Results Eighteen studies were eligible. Although there was no significant difference in the overall ADR (OR $=1.03$, 95\% CI: $0.89-1.18, \mathrm{P}=0.73$ ) between the groups with or without simethicone, a significant effect of simethicone for small adenomas $(<1 \mathrm{~cm})$ on the $\mathrm{ADR}$ was revealed $(\mathrm{OR}=2.36 ; 95 \% \mathrm{CI}$, $1.79-3.10 ; \mathrm{P}<0.00001)$. The subgroup analyses showed the beneficial effects of simethicone in the ADR among Asian populations $(\mathrm{OR}=1.45,95 \% \mathrm{CI}=1.12-1.87, \mathrm{P}=0.005)$ and

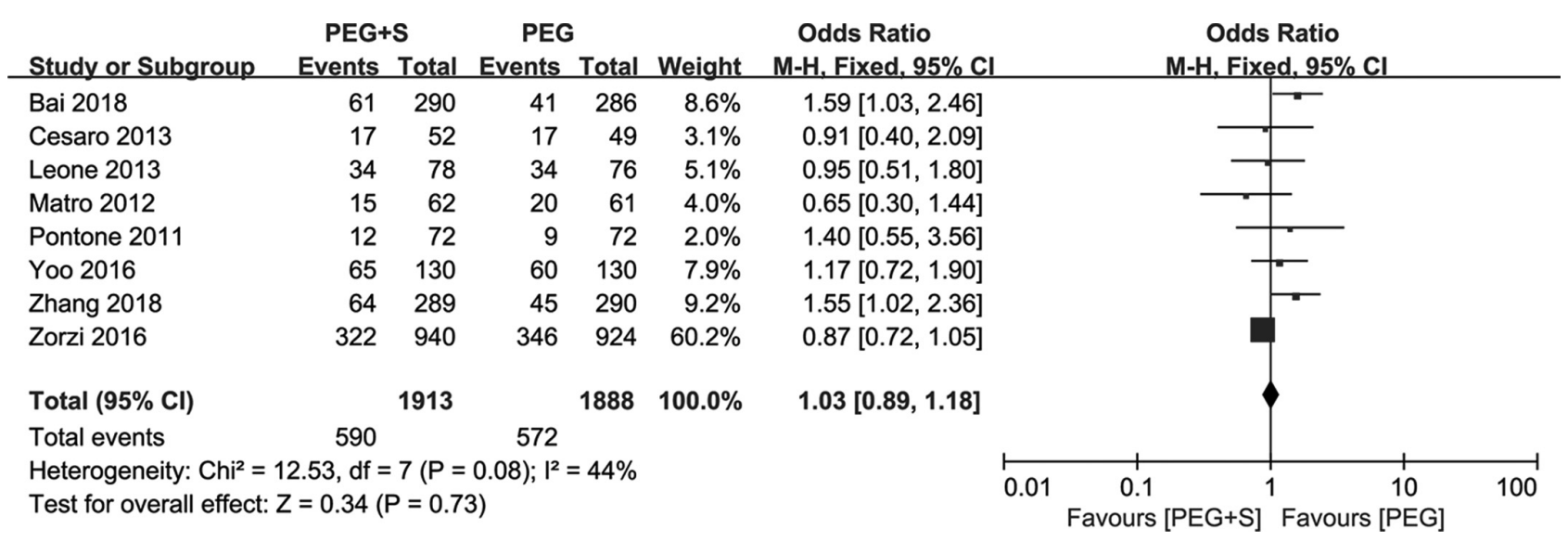

Abstract IDDF2019-ABS-0035 Figure 1 a) Forest plot of the effect of simethicone on overall ADR 
the baseline $\mathrm{ADR}<25 \%(\mathrm{OR}=1.55,95 \% \mathrm{CI}=1.16-2.07$, $\mathrm{P}=0.003)$. Moreover, there was a significant difference in the right-side ADR $(p=0.002)$ and borderline statistical significance in the left-side ADR $(p=0.04)$ between the two groups. In addition, adding simethicone to the PEG solution improved bowel preparation and patient acceptability. (figure 1a)

Conclusions In summary, we believe that the addition of simethicone to PEG is a promising bowel preparation method for colonoscopy in clinical practice among Asian populations. Additional large clinical trials are necessary to validate our results.

\section{IDDF2019-ABS-0038 PET-CT ENTEROGRAPHY CAN HELP IN PREDICTING A CLINICALLY SIGNIFICANT DIAGNOSIS IN PATIENTS WITH ILEO-CECAL THICKENING}

Abhi Kumar Singh*, Pankaj Gupta, Rajender Kumar Basher, Kaushal Kishor Prasad, Harshal Surendra Mandavdhare, Usha Dutta, Vishal Sharma. Postgraduate Institute of Medical Education and Research, India

\subsection{6/gutjnl-2019-IDDFabstracts. 123}

Background Ileo-cecal thickening is a common, nonspecific imaging finding with often uncertain significance. Role of Positron emission tomography with $18 \mathrm{~F}$-fluorodeoxyglucose integrated with computed tomographic enterography (FDG-PETCTE) in patients with ileo-cecal thickening is unclear.

Methods Consecutive patients with ileo-cecal thickening on USG from July 2017 to October 2018 underwent FDG-PETCTE followed by colonoscopy. They were divided in to two groups-those with clinically significant diagnosis (intestinal tuberculosis, Crohn's disease, infectious colitis, malignancy) and those with clinically insignificant diagnosis (normal colonoscopy or nonspecific terminal ileitis); Standardized uptake value (SUV)-max values from the ileo-cecal area were compared with the final diagnosis and area under the receiver operating characteristic (AUROC) was made to determine the best sensitivity and specificity to predict a clinically significant diagnosis.

Results Thirty-four patients were finally included, 23(67.6\%) were males and the mean age was $40.44 \pm 15.40$ years. The clinically significant diagnosis was made in $27(79.4 \%)$ patients whereas $7(20.6 \%)$ patients had a clinically insignificant diagnosis. Presence of mural thickening on CTE was present in $27(79.4 \%)$ patients. The mean SUVmax for cecum was found to be $5.68 \pm 4.09$, that for the terminal ileum was $4.19 \pm 2.52$ and for ileo-cecal valve was 4.62 \pm 3.00 . The region with the highest SUVmax value was taken for the purpose of comparisons and labelled as SUVmax (ICT). The mean SUVmax (ICT) for patients with clinically significant diagnosis was $7.162 \pm 4.382$ while those with clinically insignificant diagnosis was $3.629 \pm 0.502(\mathrm{P}<0.001)$. The area under ROC curve of the PET SUVmax (ICT) values for prediction of clinically significant diagnosis was .741 $(95 \%$ CI, 0.581-0.901). The SUVmax (ICT) value of more than 4.50 had a sensitivity of $70.4 \%$ and specificity of $100 \%$ in predicting the presence of clinically significant diagnosis whereas the value of 3.64 had a sensitivity of $74.1 \%$ and a specificity of $57.1 \%$ for picking a clinically significant diagnosis.

Conclusions PET-CT enterography could be useful in predicting if ileo-cecal thickening is due to a clinically significant underlying diagnosis and guiding the need of colonoscopy.

\section{IDDF2019-ABS-0041 THE EFFECT OF BANANA FRUIT (MUSA SAPIENTUM) AS AN ADJUNCT TO OMEPRAZOLE IN THE MANAGEMENT OF UNINVESTIGATED DYSPEPSIA}

January Ramirez*. Quezon City General Hospital and Medical Center Department of Family Medicine and Community Health, Philippines

\subsection{6/gutjnl-2019-IDDFabstracts.124}

Background Efficacy, safety, and cost-effectiveness of banana fruit as an adjunct to Omeprazole in the management of Uninvestigated Dyspepsia, (UD.)

To determine the efficacy, safety, and cost-effectiveness of banana fruit as an adjunct to Omeprazole in the management of UD.

Methods This is a therapeutic experimental study on the effectiveness of banana fruit as an adjunct to Omeprazole in the management of Dyspepsia consulting at Quezon City General Hospital Out-Patient Clinic.

The study was conducted from December-February 2018. Out of 100 subjects, 81 were included in the study. Using the systemic sampling method, patients were assigned to either Group I-39 or Group II-42. Omeprazole $20 \mathrm{mg} / \mathrm{tab}$ once a day for 2 weeks was given to Group II, whereas, 1 piece of banana fruit 3 times a day was added to the same dose of Omeprazole in Group II for 2 weeks. Talley Dyspepsia Symptoms Questionnaire Mean Global Symptom Index Scoring was used to monitor improvement in the severity of dyspeptic symptoms.

Results There were 81 subjects who completed the study. The mean age of Group I 13 (33\%) belonged to 25-30 years old age bracket, most subjects in Group II are from the 19-24 years old age bracket, 12(29\%). Both groups had more female than male subjects, Group I 23(59\%), Group II 21(50\%). Epigastric Pain was the predominant complaint on both groups with a mean of 10.08 in Group I and mean of 9.23 in Group II. As to the resolution of symptoms, Group II subjects had their symptoms resolved by the 3rd and 7th day of follow-up regardless of the MGSI score as compared to Group I subjects whose symptoms on day 7th of follow-up. Treatment in both groups was proven effective based on the resolution of symptoms within the treatment period; however, there was a faster resolution of symptoms in Group II with a mean day of 3.35 compared to 4.23 of Group I. No adverse effects were observed in both groups.

Conclusions Banana fruit as an adjunct in the treatment of UD was proven to be effective, safe and cost-effective.

\section{IDDF2019-ABS-0043 COMPARISON OF TWO METHODS FOR ENDOSCOPIC FULL-THICKNESS RESECTION OF GASTROINTESTINAL LESIONS USING OTSC}

Wenhai Wang*, Peng Li, Ming Ji, Yongjun Wang, Shengtao Zhu, Lihua Liu, Shutian Zhang. Department of Gastroenterology, Beijing Friendship Hospital, Capital Medical University, China

\subsection{6/gutjnl-2019-IDDFabstracts. 125}

Background The aim of this study was to compare and analyze the feasibility and safety of two methods of endoscopic full-thickness resection(EFTR) for the management of challenging epithelial and subepithelial neoplasms that are not amenable to resection techniques.

Methods This was a retrospective case series study of patients underwent two methods of EFTR, either of which was 